\title{
The epistemology of biomimetics: the role of models and of morphogenetic principles
}

to appear in Perspectives on Science.

\section{Ulrich Krohs}

Department of Philosophy, Westfälische Wilhelms-Universität Münster, Domplatz 23, 48143 Münster, Germany

ulrich.krohs@uni-muenster.de

\begin{abstract}
Form follows function. But form does not follow from function. It is not derivable from the latter. For realizing a desired technical function, first of all a form needs to be found that is able to realize it at all. Secondly the question arises whether an envisaged form realizes the function in a good way. Functions are multiply realizable - various different forms can bear the very same function. One needs to find a form of a technical artifact that realizes an envisaged function sufficiently efficient, robust, or whatever criteria might be imposed. This paper scrutinizes into biomimetics as one way to find a good solution to the realization problem. Drawing on an approach from the philosophy of simulations, it reconstructs the biomimetic relation as being mediated by a theoretical model. It is shown that robustness of the functioning system is usually reached in different ways in biological and in technological systems, which explains differences in morphogenetic mechanisms or principles found these fields. This reconstruction helps understanding problems with robustness in synthetic biology that occur when technical design principles are implemented in a biological system. The mimetic relation between the biological and the technical realm turns out to be asymmetric.
\end{abstract}

Keywords: Biological Function; Evolution; iGEM; Morphogenesis; Robustness; Simulation; Systems Biology; Technical Function.

\section{Introduction}

Form does not follow from function. It is therefore not a trivial task to find a form that realizes a desired technical function. "Form" in the wide sense as it is used here is not restricted to morphology and geometric properties, but including all kinds of structural relations, be they static or dynamic. In this sense, the architecture of a gene regulatory network and its regulatory relevant feed-forward and feedback loops 
count as structure, as do the architecture of a computer program and the reaction network of a chemical oscillator. "Function" on the other hand, is either the capacity realized by this structure or form, or the contribution of a component of the structure to higher capacities, in the field of biology often the contribution to keeping the integrity of the organism or more generally to its living. For the present purpose, this informal, intuitive characterization of the highly disputed concept should suffice. ${ }^{1}$

Realizations of a large number of technically interesting functions can be found in living nature, from lightweight structural elements via self-cleaning surfaces to strategies and control of locomotion on land, in water and in air. Biomimetics takes such biotic realizations of functions as antetypes for their technical realization and thus counts on living nature in finding solutions to the realization problem.

The aim of my paper is to better understand the epistemology of biomimetics. In following this aim, I first reconstruct the process of copying a biological function as being mediated by an abstract model that links the biological and the technical system (sec. 1). Next, I reflect on the sources of robustness in biological and technical systems, respectively, in order to spot a major difference between what is sometimes called morphogenetic principles in biology on the one hand, and classical engineering strategies on the other (sec. 2). Next I discuss the prospects, and an example, of mimicking not only biological structures, which always are the results of evolution and development, but also those very mechanisms (sec. 3). Finally, I discuss an example from synthetic biology that exemplifies what I want to call the mimetic asymmetry: transferability between biological and technological systems is by and large a one-way system. Therefore, one better follows a biomimetic rather than a technological approach even when re-constructing gene regulatory networks for their technical use (sect. 4).

\section{Biomimetics: models bridging biology and technology}

Copying of a biological function is an indirect process of re-constructing functionally crucial properties (static and dynamic ones) of the underlying biological structure in a technical artifact so that the mimetically re-constructed structure can fulfill the desired function in the system it is embedded in. A lotus effect coating, e.g., copies

\footnotetext{
${ }^{1}$ For the extended philosophical debate on the concept of function see (Wouters 2003; Perlman 2004; Krohs 2009a; Garson 2016).
} 
elements of the surface structure of the lotus petals, but it serves its function only in an appropriate environment in which water dabbles the surface from time to time.

The transfer from the biological to the technical realm is not only a constructive, but also an epistemic process. In order to be able to transfer the function, one needs to understand how the biological structure realizes the function (Nachtigall 2010). Uncovering "the general principles behind its functioning" (Vincent et al. 2006) is an important step in the transfer process. Biomimetics thus does not primarily aim at copying a biological structure, but at realizing a function. Copying - usually with major modifications - a biological structure is just a means to this end. It is a promising means because the biological system to be copied is itself a realization of the function.

Similarity of the biological template, the donor system, and the technical device, the receiver system, would not sufficiently guide the transfer process. If a function is realized biologically by, say, a proteinaceous structure or a polysaccharide structure, it will not usually be helpful to find a chemical realization that is similar to these often perishable - materials. In contrast, realizations might be based on metal parts, ceramic nanoparticles, or inorganic complexes. Scale might be different, as it is often the case in locomotive devices like wings or walking machines, energy supply might rely on completely different sources like batteries instead of metabolism, resulting in the need for completely different actuation systems, etc. In result, the technical system which is most similar to the biological one will usually not be the best technical implementation of the function - if it implements it at all. So another guideline than just structural similarity needs to guide the mimetic process, namely, as already quoted, an "understanding" of functioning or of its principles.

"Understanding" in this context requires that a theoretic explanation is given of how the function is realized, or of why the structure is able to perform the function. One needed to find out that the lotus effect is a consequence of superhydrophobicity which is due to the microstructure of the surface of the lotus petal in order to be able to develop lotus effect surfaces (Barthlott \& Ehler 1977, pp. 445-446; Solga 2007 et al.); that the flapping flight of many insects, birds and bats depends on high degrees of torsion of the wings before artificial insects could be constructed (Shyy 2016; Dickinson 2016; Chin 2016; Karásek 2018); that Gecko adhesion is brought about by short-ranged forces between the surface they stick onto and the minime soft branchings of the lamella of the Gecko foot, the spatulae, which nestle to the surface on the atomic scale (Bhushan 2016; Kroner \& Arzt 2016). Such an explanation comes usually in form of a model of performing the function that specifies the contributions of all components and the ways how these contributions in combination bring the function about. Biologists construct models of bird flight, of biped 
locomotion, of protein-biosynthesis, of echolocation in bats, of the lotus effect and of Gecko adhesion. These models rather than simple similarity criteria inform and direct the technical implementation of the functions at stake.

The principles of functioning of the donor system thus are depicted in an explanatory model. In functioning according to the same principles, the technical structure as the receiver system simulates the biological one. I therefore reconstruct biomimetic copying, or biomimesis, in terms of model based analog simulation (Krohs 2008): In an initial step, the biological realization of the function is modeled theoretically, i.e., understanding is gained of the structural means which realize the biological function: how the surface of the lotus flower repels dirt, how the bumblebee flies, etc. In the second step, the insights of this model are transferred to the technical realm: A technical structure is designed that satisfies the theoretical model by realizing the identified crucial structural elements - in a system that provides an appropriate context for fulfilling the desired function.

On the coarse-grained level of description, the process of model-mediated biomimesis consists of four steps:

1. Identification of a biological function or of a desired technical function in the biological realm

2. Describing how this function is realized, i.e., constructing the model that explains the biological function.

3. Envisaging an alternative, technically feasible realization.

4. Realizing the function technically.

Steps 2 and 3, often also 4, usually need to be reiterated before a satisfying result is obtained. It is also possible to further split the steps in smaller ones. However, a finer grained scheme would be valid for the particular case or for a certain class of cases onlx, so I do not include them in the general scheme. But even the coarse grained four-step-scheme is not obligatory. Research and development are fundamentally influenced by certain kinds of epistemic luck (Pritchard 2005), by path dependencies (Peacock 2009), by the order in which information is gained or retrieved, etc. This might lead not only to variation in the reiteration of steps, but even to skipping a step: It is epistemically possible that somebody is luckily envisaging a technical realization of a biological function without having an explicit model of this function. The status of the coarse grained scheme could thus best be regarded as a rational reconstruction, or as an in principle-reconstruction, of the process of the development 
of biomimetic technology. It is certainly not the only possible reconstruction, but, as is shown below, one that helps understanding, and consequently also planning and steering, the process.

I therefore strongly disagree with the judgment that the epistemological framework of biomimetics has only one part that can be fixed, namely "searching biological literature for functional analogies to implement" (Vincent et al. 2006). The central steps of the epistemic process do concern modeling rather than library work. They involve generation and re-application of the model of how the biological system implements the function. Literature search is a way to retrieve the model and is thus pragmatically often highly important. But this means only that one relies on model building that was done by others before and does not change the general epistemic process. It can also be done by others in an interdisciplinary. As Green et al. (2019) point out, biomimicry practitioners, typically engineers, physical scientists, chemistry and biological engineers, may often profit from direct interaction with those knowledgeable about the wealth of functions realized by biological traits, i.e., with biologists in natural history museums an collections. Certainly, other sources and cooperation partners from biology will as well be able to contribute also directly, not only via their publications being retrieved by the biomimeticists. The need for interdisciplinary co-operation can also be read from the four-step scheme given above. Step 1 relies on empirical description and research and is thus primarily a task for empirical biologists, step 2 on the application of - often formal - modeling techniques, done often by theoretical biologists. Steps 3 and 4 are demanding the core expertise of engineers and perhaps further co-operation with physicists, chemists, or biologists.

\section{Robustness in biological and in technical systems}

Biological systems persist under changing conditions, and they adjust to these conditions. They are stabile enough to resist thermodynamic, mechanical and other challenges. On the other hand, they must not be too stabile because this would prevent adjustments. Persisting by means of adjustment to changing conditions involves changes in structure and operation of the system. Maintained is not an exact form, but the organizational integrity and the self-regulatory processes as well as those linked to them. A system adjusting in such a way is called a robust system. "Robustness is the ability of a system to maintain its functionality across a wide range of operational conditions" (Hammerstein et al. 2006). This definition avoids reference to specifically biological criteria for robustness and thus takes into account that robustness does not only hold of biological, but often also of technical systems. Robustness of technical artifacts, however, differs significantly from robustness of 
biological organisms. This difference has important consequences for biomimetics. In order to discuss these, we need first to compare aspect or kinds of robustness in both sorts of entities. I am focusing only on aspects of particular importance for the epistemology of biomietics.

Let us first look at biological organisms. They need to be robust in several respects. Since organisms are not very stabile in the classical sense - they can be wounded, poisoned, damaged by heat, etc. - they need repair mechanisms. Since many organisms feed on different sources, they also need mechanisms for switching their metabolism. And since challenges vary during the day and during the year, they need to follow this external rhythm and respond differently to the very same kind of stimulus at different times. In addition, we the environment challenges not only the fully developed organism, but also its development. So development needs itself to be robust and to lead to the same, or at least: to similar results, independently of deteriorations.

Besides such external challenges, there are also internal ones: The genetic material is flexible and subject to mutations. So developmental pathways need to be robust with respect to mutations. This is achieved in part by a degenerated code, but more specifically by the structure of the gene regulatory networks that govern development. These networks provide redundant functionality and regulation mechanisms so that blocking or altering a node or link in the network by mutation usually does not affect its overall action.

Robustness also holds for lineages on an evolutionary scale. Lineages remain stabile despite changing environments and despite evolutionary processes going on - in fact, they often remain stabile due to evolutionary processes. Robustness can be achieved by keeping variation even under selection so that future generations adapt quickly. So the fundamental mechanisms that make organisms robust are the following:

a) Mechanisms of generation that lead in a robust way to a robust result;

b) Mechanisms of maintenance, incl. replacement;

c) Mechanisms of adjustment.

For details and further mechanisms of robustness see Hammerstein et al. (2006). Specific accounts of overall robustness are given in the theory of autopoietic systems (Maturana \& Varela 1980) and by Rosen's (M,R)-systems (Rosen 1973; 1991; cf. Letelier et al. 2006). 
This means that an organism is undergoing almost permanent modification. It adjusts to the environment as well as to internal modifications. It thus can be said that robustness in biological organisms is brought about by ongoing morphogenesis.

Robustness in technology is usually achieved by different means. First of all, measures are often taken that reduce wear and tear of the artifact. This means that robustness in technology is much more based on stability and much less on a regular turnover of components, which turned out to be most important in the biological case. Another aspect of robustness in technical systems is a modular architecture that helps to maintain the system (besides its advantages in development and in construction). If and component becomes damaged, the damage is often isolated in the module it occurs in, so that no major problem occurs until this module can be replaced. Modularity in biology, in contrast, might often be less relevant for (self)maintenance of the system than for development and evolution. Moreover, modules e.g. of gene regulatory networks are usually much more interlinked and even overlapping and delineation of modules is often more an epistemically valuable constructive step rather than a neutral depiction of a complex network (Krohs 2009b).

So while biotic systems gain robustness through adaptive fluidity, technical systems are often robust because of stability, elasticity or stiffness, i.e., the ability to return to the previous state after deterioration. Before enquiring consequences of this difference, I am now looking at the prospects of transferring strategies for robustness from biology to the technical realm.

\section{Biomimetic approaches to morphogenesis}

If robustness is important for technical devices, including biomimetic ones, and if the biological processes of evolution and development lead to robust biological structures, it seems advisable to take advantage of the capacities of these biological processes and to include them in the biomimetic approach. Definitions or characterizations of biomimetics quite often include the idea of mimetic construction, in addition to mimetic structure and function. Copying synthetic pathways is sometimes even considered being on a par with copying structure and function: "biomimetics is the study of biological structures, their function, and their synthetic pathways, in order to stimulate and develop these ideas into synthetic systems similar to those found in biological systems." (Sarikaya 1995, p.xi). It might nevertheless be useful to discern different levels of technical biomimesis. According to Borsari, the first level is copying biological structures in order to realize a function technically. Examples are the lotus effect and gecko adhesion. The second level refers to 
mimicking complex strategies from nature, where fault tolerance in the brain by redundancy is mentioned as possibly providing a helpful model in electronics. The third level, then, is mimicking evolution (Borsari 2017).

While Borsari's first level includes mimicking not only structures, but also their involvement in processes, which might already be quite complex, his second level demands mimicking complex strategies. His example makes clear that this does not primarily refer to developmental processes, which also could be described as strategies to bring about the very same structure under a broad range of conditions, but rather to complex mechanisms in the fully developed biological entity that make it robust. The borderline between developmental robustness and robustness of developed entities by means of complex strategies might be fuzzy and even vanish under a certain perspective. For systematic reasons I nevertheless want to keep this distinction. Then, biomimetics of levels one and two mimicks evolved and developed biological features: level one is the mimicking of structures and their dynamics and thereby implementing a function; level two is mimicking complex strategies rather than mere processes. The difference, again, is a matter of degree, it is difficult to find a criterion for what a strategy is. What makes the difference is how it is modeled: A strategy might be best regarded as a complex, plastic process that is modeled as a problem solving strategy. With this distinction in mind, fault tolerance mechanisms in electronics, but also locomotion of artificial worms (Menciassi at al. 2004; Boxerbaum et al. 2012) and goal-finding behavior of vehicles (Braitenberg 1984) are examples of the second level of biomimetics, while the processes involved in selfcleaning of lotus effect-surfaces and reversible adhesion of gecko pads remains on level one.

Level three differs from the first two levels in focusing on the generation of form, not on the given form and the processes it is involved in. Level three deals literally with biomimetic approaches to morphogenesis. Borsari mentions evolution as the morphogenetic process to be mimicked, and this is what we find in the literature about technical mimicking of biological morphogenesis. Evolutionary strategies where propagated already in an early phase of the field of biomimetics (Rechenberg 1973). Among the examples are the evolutionary way to shape a two-phase nozzle (Schwefel 1968), the evolution of neuronal networks as controllers of autonomous robots (Hülse et al. 2004), the directed evolution of proteins (Kan et al. 2016; Arnold 2019), and the evolution of artificial cells (McCaskill 2009). As the examples show, we are often, but not always still dealing with systems close to biological ones when evolutionary strategies are at stake. Evolutionary strategies are also quite often applied in certain fields of applied mathematics and in programming. I will not consider these examples because they are dealing with abstract entities that have 
symbolic content. Including this realm would demand opening up another debate. I confine my treatment of level three biomimetics to cases of concrete entities. Results can be transferred to the abstract case afterwards.

Let me start with some general considerations. Systems realizing functions do not emerge from just mixing components. They need to be put together in a systematic way. In the biological realm, this seem to restrict possible outcomes, since these can only be results of developmental processes, and since the developmental processes can themselves only be results of evolutionary processes. So we can expect to find a nested path dependence constraining the outcomes. On the other hand, nature obviously found ways to come up with results on complicated paths that we would judge as extremely clever if found in technology, so restrictions might not be an issue in the long run.

Biomimetic artifacts, on the other hand, are not usually build in a biomimetic way. Their construction follows technical paradigms rather than morphogenetic principles learned from biology. As it turns out, it is nevertheless valuable to look at such principles and consider the third level of biomimetics, the level of morphogenesis. Since biological morphogenesis includes not only phylogenetic, but also ontogenetic processes, my take of this level is wider than Borsari's and includes both, evolution and development (Krohs 2021). This widening seems required because even one of the most intriguing examples of biomimetic evolution, the already mentioned evolutionary optimization of a two-phase nozzle (Schwefel 1968), combines evolutionary and developmental mechanisms. The nozzle with its initial taper in diameter was cut into sections so that the opening in each section had a different diameter. Sections were then put together in an arbitrary order, resulting in an irregular shape of the nozzle. Optimization then followed a scheme of variation of the order of the sections and selection according to measured efficiency. In the more efficient nozzle, two arbitrarily chosen segments exchanged against each other and again efficiency was measured. The better structure was chosen for further changes. This trial and error process of optimizing flow speed and efficiency lead to a nozzle with irregular shape that would hardly have been rationally constructed (Schwefel 1968). Since technical morphogenesis was driven by an evolutionary paradigm taken from biology, the optimization is to be taken as biomimetics of level three.

However, despite implementing variation and selection, this process of technical development is in important respects not an evolutionary process. With this caveat $\mathrm{I}$ do not refer to the fact that trial and error procedures do belong to the methodological spectrum of engineering anyway so that the distinction between a rational methodology of engineering and the trial-and-error process of evolution is overstated 
anyway (Morange 2013). At stake is instead that the engineering process, while taking up one aspect of evolution, namely variation and selection, realizes variation in a way highly different from what is found in evolution: not as variation between different individuals, but as varying again and again one and the same individual which is much more resource efficient. Similar changes of the shape of an individual by rearrangements are known from biological development. In combination with growth processes, such change by rearrangement might even be considered the kernel of a developmental process. So the "evolution" of the nozzle crosses the line between evolution and development. But also the developmental metaphor would not be fully adequate for the case of the optimization of the nozzle. Biological development is usually taken to follow either a definite order of steps, or to lead, via regulation, to a predefined result. None of both seems to hold in our case. But the spectrum of developmental mechanisms is much broader. The shape of a tree is prefixed only to a degree that leaves open a wide range of options. Branches can be ordered in this or another way, as do leafs, as do roots. Interaction with the environment - with wind or with stones in the soil, e.g. - has a huge impact on the precise morphology or the tree. But also clear-cut changes of position occur. Cells migrate to their "destination" in the embryo, morphogenetic factors are spreading by diffusion, the differentiation of tissues is induded by another tissue that comes into contact with it due to growth of some third part of the embryo, etc. (Gilbert 2010). Though it is quite obvious that Schwefel's experiments with the nozzle were inspired by evolution rather then by biological development, we can hardly tell it a clear-cut case of an evolutionary procedure.

Fortunately, nothing depends on such a classification. The only reason why I insist in the different aspects of evolution and development in biological morphogenesis is that obviously the pool of morphogenetic mechanisms is much larger than only some trial-and-error aspect of evolution. Biomimetics of level three could draw not only on this single aspect, but on a wide spectrum of mechanisms. Many of them have evolutionary as well as developmental roots. Developmental mechanisms did themselves evolve, and evolutionary mechanisms do not bring about isolated, fully developed traits, but traits as the result of developmental processes. Realizable development is a precondition of evolution, at least of eukaryotes. Development makes organisms even susceptible to environmental influence on evolution, namely via environmentally induced modifications of developmental pathways (Gilbert 2010), which is accounted for in the so-called extended synthetic theory of evolution (cf. Sultan 2015; Huneman \& Walsh 2017).

I do not want to propose that biomimetics should try to mimic this entanglement of evolution and development. I just want to point out that there is much to discover in 
evolution and development that might be worth mimicking in level three biomimetics. An example might be the development of bones. When a bone grows, its microstructure develops in direct response to the mechanical stress it is submitted to, in a way that allows for maximum stability with respect to the forces that are actually imposed on it, by minimal weight. This is mimicked in the construction of bone repair materials (Zhang et al. 2014; Chahal et al. 2019), in a field of biomimetics that has itself biological application.

\section{The mimetic asymmetry}

Having reconstructed in sec. 1 the biomimetic relation that holds between biological and technical systems as mediated by a model, it looks as if transfer could go both directions so that a biological - better to say: biotechnological - system designed by a technomimetic approach would be as easy to conceive as biomimetic classical technology. However, it needs to be taken into account that technical morphogenetic principles differ from biotic ones in several respects. Ontogenetic biological morphogenesis is based on developmental processes. The structures brought about by such process are constrained by the requirements that mechanisms are available that bring such a process about, that any intermediate state of the process is viable, and that the processes is robust. Technical construction, while not being restricted by developmental pathways, underlies its own constraints of technical feasibility, budgeting, etc. Phylogenetic morphogenesis in the realm of biology again underlies constraints resulting from path dependence and other historical contingencies, while analogous processes in technical development can easily bring together disparate paths. Also the stability requirements for the resulting structures are different in the biological and in the technical realm.

Despite these differences, considerations about the symmetry of the transfer between biology and technology are at the basis of several projects in synthetic biology. Synthetic biology is a heterogeneous field, including top-down modification of existing organisms as well as bottom-up approaches to the de novo synthesis of new life forms from nonliving materials, and rational engineering as well as evolutionary approaches (Krohs \& Bedau 2013). I shall be dealing only with rational engineering in the modification of existing organisms and focus on the approach that lead to the idea of programming cells by arranging bio-bricks, which are short pieces of DNA with particular functions, in a way similar to writing a computer program (Endy 2005). The idea is to generate a particular behavior of the cell - be it a recurrent sequence of states, e.g., in an oscillator like a circadian clock, or an artificial biosynthetic pathway. This approach is often considered to be as appealing as its success is poor. The annual student International Genetically Engineered Machine 
competition iGEM, which is based on it, succeeds for other reasons than for the development of stabile biotic machines (cf. Betten et al. 2018), and right so.

The technomimetic aspect of this approach is quite clear, and stated explicitly by Endy and others. A flow chart is translated into a modular gene sequence in order to generate a simple gene regulatory network. The program is kept as linear as possible, following a strategy from modular programming in computer science. It links linear sequences with decision points, loops, and plugged-in subroutines. As in rational engineering, components are standardized, and modules as decoupled from each other as much as possible. However, it usually turns out that the result is unstable. Elements are expelled from the program, the chassis cell does not copy the program - the engineered DNA - as expected. How and why does this happen? The "how" is obvious: as always in genetic engineering, the engineered cells need to divide and multiply in order to generate a significant, even a measurable output. As soon as cell division comes into play, all kinds of modifications of the genetic material may happen, including loss of genetic material. Measures might be taken to minimize this, like coupling to antibiotic resistance genes, but in the end multiplication of engineered cells happens always in a way that allows for evolutionary change.

The observation is thus that the genetic material of the engineered cells is evolutionary unstable, as it was already the case in the repressilator, a forerunner and still best-known example of this approach (Elowitz \& Leibler 2000). Evolutionary instability corrupts the technomimetic bio-brick approach. But why is it so, despite the success of the rational engineering approach in many other fields of application? The answer I want to propose refers to the conditions for robustness of biological and of technological devices, respectively, as reconstructed in sec. 2. There, we have seen that robustness in biological systems is based in ongoing morphogenesis and in a deep entanglement of all processes: structures are not stabile, but permanently rebuilt and adjusted, by trial and error and/or by regulated replacement of all components, and processes are entangled by involving components that at also contribute to other processes. In technical systems, on the other hand, robustness is based in low rates of wear and tear, in decoupling and other mechanisms limiting the impact of errors, and in easy maintenance. Though there are certainly exceptions, robustness of biological systems lies in general in the interdependence of all their components, while robustness of technical systems is based in the separation of effects, even though functions are brought about only by coupling and interaction. We have messy, holistic interaction leading to robustness in biological systems, and sterile, analytic interaction leading to robustness in technical systems. 
This observation suggests a plausible answer to the question why a rationally planned, modified organism lacks robustness: the implanted modules are not deeply embedded in the system of the organism, but added in a sterile way. But the modified organism cannot be maintained like a technical artifact, which would allow for robustness of such a delicate system with only few interconnections. It maintains itself - resulting in expelling every implant that is not deeply embedded in the functioning of the cell. Put in evolutionary terms: rationally planned top-down synthetic biology tries to get the implemented genetic programs selected, and the strategy is to couple them superficially to other genes that are under selection pressure. The setting selects for these other genes, and by coupling this results in selection of the implanted program. So far the rational. In reality, selection of (Sober 1984) the implanted program is not sufficient, since the coupling to the genes selected for is easily dissolved in a few evolutionary steps. The technomimetic biological system is still a biological system and underlies biological - evolutionary - criteria of robustness. Seen this way, expelling the implanted material is a result of the robustness of the cell rather than an indicator of lacking robustness.

This situation could, in principle, be solved by applying a biomimetic approach of the third level: by taking advantage of evolutionary and developmental mechanisms in stabilizing the genetically engineered system. This would require deeply embedding the added components rather than implanting them in a decoupled way. The goal would be that selection for these components occurs, e.g. because of their involvement in other than the constructed pathways, rather than mere selection of them. The components need to perform roles in fundamental processes within the cell. I do not dare speculating whether this is possible at all. Strategies would be required how such additional genes could be embedded deeply into metabolism. These strategies might best be copied from biological processes. They will almost certainly be far away from the present strategy of rational construction.

The following picture for a biological cell with its gene regulatory network may illustrate my claim: Look at the cell and its metabolism as if it were a bowl filled with pebbles. The pebbles build up a fairly stabile structure since they are supporting each other and are put in form by the bowl. Implementing additional genes would be like adding marbles on top of the pebbles. This will work for a few added marbles, in particular if you arrange them in a single layer or in the shape of a pyramid. Now try to build a delicate sculpture, say with the proportions of a Giacometti, from the added marbles, with as few links to the pebbles as possible, which the aid of only some viscous grease. It is highly unlikely that your structure will survive for a longer time and remain intact when moving or shaking the bowl. A much better way to stabilize the marble structure would be to embed it in the pebbles. It might be not so 
easy and you will not easily be able to see it any longer, but it is there and it is stable - stabilized by numerous interactions with the pebbles. If the delicate sculpture stands for the sophisticated program constructed out of bio-bricks, and moving and shaking of the bowl stands for selection pressure, this picture gives a pretty good understanding of the lack of robustness of the genetically engineered "machine" cell and for the problems stabilizing it - which hold on as long as the add-ons are not deeply embedded in the fundamental processes of the cell.

We now can describe the biomimetic relation between biological and technical system more detailed than in sec. 1. Instead of the biological and the technical system being just two realizations of a model of the desired function, which lead to a symmetrical relation which turned out not to hold in the example from synthetic biology, the picture needs to account as well for robustness. A biomimetic device needs to be a robust implementation of the model, where robustness is to be achieved in the way adequate for the receiver system, not for the donor system. If the receiver system is a purely technical one, criteria for technical robustness hold. If it is a biological system, biological robustness needs to be implemented. This means that the mimetic relation is doomed to fail when robustness of the receiver system is constructed according to robustness mechanisms of the donor system. I have shown these for the technomimetic reconstruction of a biological system, but it will hold also in the other direction - which simply does not attract attention because it is observed routinely anyway: a biomimetic artifact needs to be robust in terms of artifact robustness rather than implementing biological robustness (however such implementation might be thought of). As long as deep embedding of new components in a biological organism is not possible, the mimetic relation is asymmetric and leads (almost) only from biological to technical systems, not the other way around.

\section{Conclusion}

Biomimetics is a relevant and very successful approach to implement technical functions. The reconstruction of the epistemology of biomimetics demonstrates that the approach is based on models of the function that link biological and technical system. On the fist glance, the relation between biological and technical system is therefore symmetrical. Since the strategies to make the systems robust differs between biology and technology, the transfer is limited by needs for robustness. In this regard, not the donor system, but the receiver system determines the criteria of a successful implementation of the function - which breaks the symmetry of the relation between donor system, model, and receiver system. This helps understanding why the bio-brick approach in synthetic biology by and large failed. It tries to 
transfer not only the implementation of the function from the technical to the biological realm, which nicely works, but also the means to achieve robustness, which failed. In a biological system, even if it is technomimetically re-engineered, robustness needs to be of the biological type.

\section{References:}

Arnold, Frances H. 2019. "Innovation by Evolution: Bringing New Chemistry to Life (Nobel Lecture)." Angewandte Chemie International Edition 58:14420-14426.

Barthlott, Wilhelm, and Nesta Ehler. 1977. "Rasterelektronenmikroskopie der Epidermisoberflächen von Spermatophyten.” Pp. 367-467 in Tropische und subtropische Pflanzenwelt. Vol. 19. Mainz: Akademie der Wissenschaften und der Literatur. Wiesbaden: Franz Steiner Verlag.

Betten, Afke Wieke, Virgil Rerimassie, Jaqueline E.W. Broerse, Dirk Stemerding and Frank Kupper. 2018. "Constructing future scenarios as a tool to foster responsible research and innovation among future synthetic biologists." Life Sci Soc Policy 14:21.

Bhushan, Bharat. 2009. “Biomimetics: Lessons from Nature - an Overview.” Philosophical Transactions of the Royal Society A 367:1445-1486.

Bhushan, Bharat. 22016. "Gecko Effect.” Pp. 1319-1328 in Encyclopedia of Nanotechnology. Edited by Bharat Bhushan. Dordrecht: Springer.

Borsari, Andrea. 2017. "Human Mimicry and Imitation: The Case of Biomimetics." Aisthesis. Pratiche, Linguaggi E Saperi dell'estetico 10:51-61.

Boxerbaum, Alexander S., Kendrick M. Shaw, Hillel J. Chiel, and Roger D. Quinn. 2012. "Continuous Wave Peristaltic Motion in a Robot." The International Journal of Robotics Research 31:302-318.

Braitenberg, Valentino. 1984. Vehicles: Experiments in synthetic psychology. Cambridge: MIT Press.

Chahal, Sugandha, Anuj Kumar, and Fathima S. J. Hussian. 2019. "Development of Biomimetic Electrospun Polymeric Biomaterials for Bone Tissue Engineering. A Review." Journal of Biomaterials Science, Polymer Edition 30:1308-1355.

Chin, Diana D., and David Lentink. 2016. "Flapping Wing Aerodynamics: From Insects to Vertebrates.” The Journal of Experimental Biology 219:920-932.

Dickinson, Michael H., and Florian T. Muijres. 2016. "The Aerodynamics and Control of Free Flight Manoeuvres in Drosophila." Philosophical Transactions of the Royal Society B 371:20150388.

Elowitz, Michael B., and Stanislas Leibler. 2000. "A Synthetic Oscillatory Network of Transcriptional Regulators." Nature 403:335-338.

Endy, Drew. 2005. "Foundations for Engineering Biology.” Nature 438:449-453.

Garson, Justin. 2016. A Critical Overview of Biological Functions. Dordrecht: Springer.

Gilbert, Scott F. ${ }^{9} 2010$. Developmental Biology. Sunderland: Sinauer Associates.

Green, David W., Jolanta A. Watson, Han-Sung Jung, and Gregory S. Watson. 2019. "Natural History Collections as Inspiration for Technology.” BioEssays 41:1700238.

Hammerstein, Peter, Edward H. Hagen, Andreas V. M. Herz, and Hanspeter Herzel. 2006. "Robustness: A Key to Evolutionary Design." Biological Theory 1:90-93.

Huneman, Philippe, and Denis M. Walsh (eds.). 2017. Challenging the Modern Synthesis: Adaptation, Development, and Inheritance. Oxford: Oxford University Press.

Hülse, Martin, Steffen Wischmann, and Frank Pasemann. 2004. "Structure and Function of Evolved Neuro-Controllers for Autonomous Robots." Connection Science 16:249-266.

Kan, S. B. Jennifer, Russell D. Lewis, Kai Chen, and Frances H. Arnold. 2016. "Directed Evolution of Cytochrome C for Carbon-Silicon Bond Formation: Bringing Silicon to Life." Science 354:10481051. 
Karásek, Matěj, Florian T. Muijres, Christophe De Wagter, Bart D. W. Remes, and Guido C. H. E. de Croon. 2018. "A Tailless Aerial Robotic Flapper Reveals that Flies Use Torque Coupling in Rapid Banked Turns.” Science 361:1089-1094.

Krohs, Ulrich. 2008. "How Digital Computer Simulations Explain Real-World Processes." International Studies in the Philosophy of Science 22:277-292.

Krohs, Ulrich. 2009a. "Functions as Based on a Concept of General Design.” Synthese 166:69-89.

Krohs, Ulrich. 2009b. "The Cost of Modularity." Pp. 259-267 in Functions in Biological and Artificial Worlds: Comparative Philosophical Perspectives. Edited by Ulrich Krohs and Peter Kroes. Cambridge, Mass: MIT Press.

Krohs, Ulrich. 2021. "Evolution und Entwicklung - universelle Konzepte?” Pp. 77-90 in Biologische Transformation - Interdisziplinäre Grundlagen für die angewandte Forschung. Edited by Tomas Marzi, Hans-Werner Ingensiep and Heike Baranzke. Oberhausen: Laufen.

Krohs, Ulrich, and Mark A. Bedau. 2013. "Interdisciplinary Interconnections in Synthetic Biology." Biological Theory 8:313-317.

Kroner, Elmar, and Eduard Arzt. ${ }^{2}$ 2016. "Gecko Adhesion.” Pp. 1308-1319 in Encyclopedia of Nanotechnology. Edited by Bharat Bhushan. Dordrecht: Springer.

Letelier, Juan-Carlos, Jorge Soto-Andrade, Flavio Guíñez Abarzúa, Athel Cornish-Bowden, and María Luz Cárdenas. 2006. „Organizational Invariance and Metabolic Closure: Analysis in Terms of (M,R) Systems.” Journal of Theoretical Biology 238:949-961.

Maturana, Humberto R., and Francisco J. Varela. ${ }^{2}$ 1980. Autopoiesis and Cognition: The Realization of the Living. Dordrecht: Reidel.

McCaskill, John S. 2009. PACE Report: Programmable Artificial Cell Evolution. http://www.biomip.org/pacereport/the pace_report/index.html

Menciassi, Arianna, Samuele Gorini, Giuseppe Pernorio, Liu Weiting, Francesco Valvo, and Paolo Dario. 2004. "Design, Fabrication and Performances of a Biomimetic Robotic Earthworm." Paper presented at the 2004 IEEE International Conference on Robotics and Biomimetics, August 2226.

Morange, Michel. 2013. "Comparison Between the Work of Synthetic Biologists and the Action of Evolution: Engineering Versus Tinkering.” Biological Theory 8:318-323.

Nachtigall, Werner. 2010. Bionik als Wissenschaft. Erkennen - Abstrahieren - Umsetzen. Berlin: Springer.

Peacock, Mark S. (2009). "Path Dependence in the Production of Scientific Knowledge." Social Epistemology 23:105-124.

Perlman, Mark. 2004. “The Modern Philosophical Resurrection of Teleology.” The Monist 87:3-51.

Pritchard, Duncan. 2005. Epistemic Luck. Oxford: Oxford University Press.

Rechenberg, Ingo. 1973. Evolutionsstrategie - Optimierung technischer Systeme nach Prinzipien der biologischen Evolution. Stuttgart: Frommann Holzboog.

Rosen, Robert. 1973. “On the Dynamical Realization of (M, R)-Systems.” Bulletin of Mathematical Biology 35:1-9.

Rosen, Robert. 1991. Life Itself: A Comprehensive Inquiry Into the Nature, Origin, and Fabrication of Life. New York: Columbia University Press.

Sarikaya, Mehmet, and Ilhan A. Aksay (eds.). 1995. Biomimetics: Design and Processing of Materials. Woodbury: AIP Press.

Schwefel, Hans-Paul. 1968. "Experimentelle Optimierung einer Zweiphasendüse.” Report HE/F 35-B from AEG Forschungsinstitut Berlin.

Shyy, Wei, Chang-kwon Kang, Pakpong Chirarattananon, Sridhar Ravi, and Hao Liu. 2016. "Aerodynamics, Sensing and Control of Insect-Scale Flapping-Wing Flight." Proceedings of the Royal Society A 472:20150712. 
Sober, Elliott. 1984. The Nature of Selection: Evolutionary Theory in Philosophical Focus. Cambridge, Mass: Bradford/MIT Press.

Solga, Andreas, Zdenek Cerman, Boris F. Striffler, Manuel Spaeth, and Wilhelm Barthlott. 2007. "The Dream of Staying Clean: Lotus and Biomimetic Surfaces." Bioinspiration \& Biomimetics $2: 126-134$

Sultan, Sonia E. 2015. Organism and Environment: Ecological Development, Niche Construction, and Adaptation. Oxford: Oxford University Press.

Vincent, Julian F. V., Olga A. Bogatyreva, Nikolaj R. Bogatyrev, Adrian Bowyer, and Anja-Karina Pahl. 2006. "Biomimetics: Its Practice and Theory." Journal of the Royal Society Interface 3:471482.

Wouters, Arno G. 2003. "Four Notions of Biological Function." Studies in History and Philosophy of Biology and Biomedical Science 34:633-668.

Zhang, Xuesong, Ming Lu, Yan Wang, Xiaojing Su, and Xuelian Zhang. 2014. "The Development of Biomimetic Spherical Hydroxyapatite/Polyamide 66 Biocomposites as Bone Repair Materials." International Journal of Polymer Science 2014:579252. 